
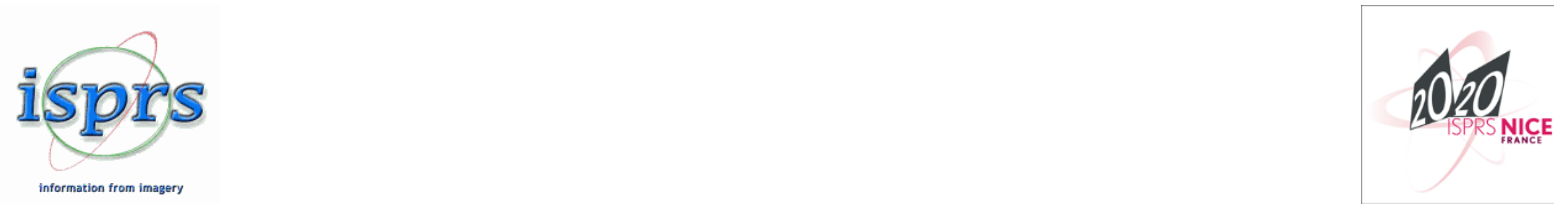

\title{
Preface
}

\section{Technical Commission I}

For ISPRS Technical Commission I (TC I), a remarkable number of 189 submissions for the 2020 Congress edition of ISPRS Annals and ISPRS Archives was received. This included both full paper and abstract submissions from all over the world. Encouraged by the success of double blind paper reviewing in preparation of the 2016 Prague congress and the 2018 Karlsruhe symposium, also this time, TC I organized a strict peer-reviewing process. This included double-blind reviewing for full papers as well as a two-stage evaluation of abstract submissions - first stage based on the submitted abstracts ("conditional acceptance"), and second stage after submission of the respective final papers.

In total, we received 69 full paper submissions and 120 abstract submissions, which indicates a nice trend towards full paper submissions compared to previous TC I events.

45 full papers passed the double-blind peer-review process and were accepted for publication in the ISPRS Annals (acceptance rate 64\%); usually, three or four reviews were obtained for each paper. Abstract submissions and their respective final papers were reviewed by a team of professionals. In total, 76 papers were accepted to the ISPRS Archives.

The manuscripts in both the ISPRS Annals and Archives cover a broad range of topics related to remote sensing platforms, technologies, systems and related methods and reflect the current trends in algorithmic research and developments in sensing and data acquisition methods. Noteworthy is that numerous contributions were submitted to Intercommission WGs of TC I with TC II and TC IV, which underlines the increasing trend towards an integral approach to sensors, systems and methods in photogrammetry, remote sensing and mobile mapping.

Stefan Hinz, Raul Feitosa, Martin Weinmann, Boris Jutzi 